\title{
From branch to bedside: Youyou Tu is awarded the 2011 Lasker DeBakey Clinical Medical Research Award for discovering artemisinin as a treatment for malaria
}

\begin{abstract}
he
he WHO has indicated that in 2009 , the most recent year for which data are available, approximately 250 million people were infected with malaria, and nearly one million of those patients succumbed to their infections. But without Youyou Tu (Institute of Materia Medica, Academy of Traditional Chinese Medicine and China Academy of Chinese Medical Sciences) (Figure 1), many of those 249 million others may not have survived. This is the story of how Tu led a team that transformed an ancient Chinese method of using the herb qinghao into artemisinin, the most powerful antimalarial medicine that currently exists. And as the Chinese proverb has it, "He who plants a garden, plants happiness.”
\end{abstract}

\section{History of malaria}

Malaria has likely infected humans for more than 50,000 years (1), but the first written reference to the stereotypical periodic fevers of malaria can only be traced back to 2700 BCE in China (2). Significant scientific studies on malaria began in the 1880s, when Scottish physician Ronald Ross proved that mosquitoes were the vector for malaria transmission and provided the complete life cycle of the malaria parasite (3). For this work, Ross received the 1902 Nobel Prize in Physiology or Medicine. The French military doctor Charles Laveran later observed parasites inside rbc from malaria patients (Figure 2) and thereby showed for the first time that a protist could underlie a disease (4). For this and later discoveries, Laveran was awarded the 1907 Nobel Prize in Physiology or Medicine.

Most human cases of malaria are caused by one of the four species of Plasmodium parasites: P. falciparum, P. vivax, $P$. ovale, and P. malariae. Despite efforts over the past century, more than 2 billion people remain at risk of contracting malaria, which primarily affects tropical areas, where higher temperatures and abundant rainfall are most suitable for the development of the malaria-carrying Anopheles mosquitoes (5). Plasmodium parasites all develop through the same general life cycle, alternating between the human and mosquito (Figure 3 ). The cycle begins when a Plasmodium-carrying female anopheline mosquito feeds on human blood. Sporozoites injected into the skin migrate via the bloodstream to the liver, where they infect hepatocytes. Over 5-16 days, each parasite grows and divides into thousands of merozoite forms that then exit from individual hepatocytes into the blood, where they infect erythrocytes. Some P. vivax and $P$. ovale parasites can remain latent in the liver until they activate and cause relapses of malaria months or years later. Blood-stage Plasmodium parasites undergo repetitive rounds of invasion, growth, and division in one day (P. knowlesi), two days (P. falciparum, P. vivax, and P. ovale), or three days (P. malariae). P. falciparum becomes most harmful in this stage, as infected host rbc avoid the spleen by sequestering in capillaries and microvenules of the brain and other vital organs (the other malaria parasite species do not do this). This stage of bloodstream infection can last for months if not treated. During this stage, some parasites leave the asexual replication phase and differentiate into male and female gametocytes. Other anopheline mosquitoes ingesting a blood meal from an infected human then mate and generate thousands of offspring sporozoite forms that travel to the mosquito salivary glands, where the sporozoites remain before initiating another life cycle (6).

The first symptoms in an infection usually occur ten days to four weeks after infection, though they can appear as early as eight days or as long as a year after infection. Most symptoms are caused by the release of merozoites into the bloodstream,

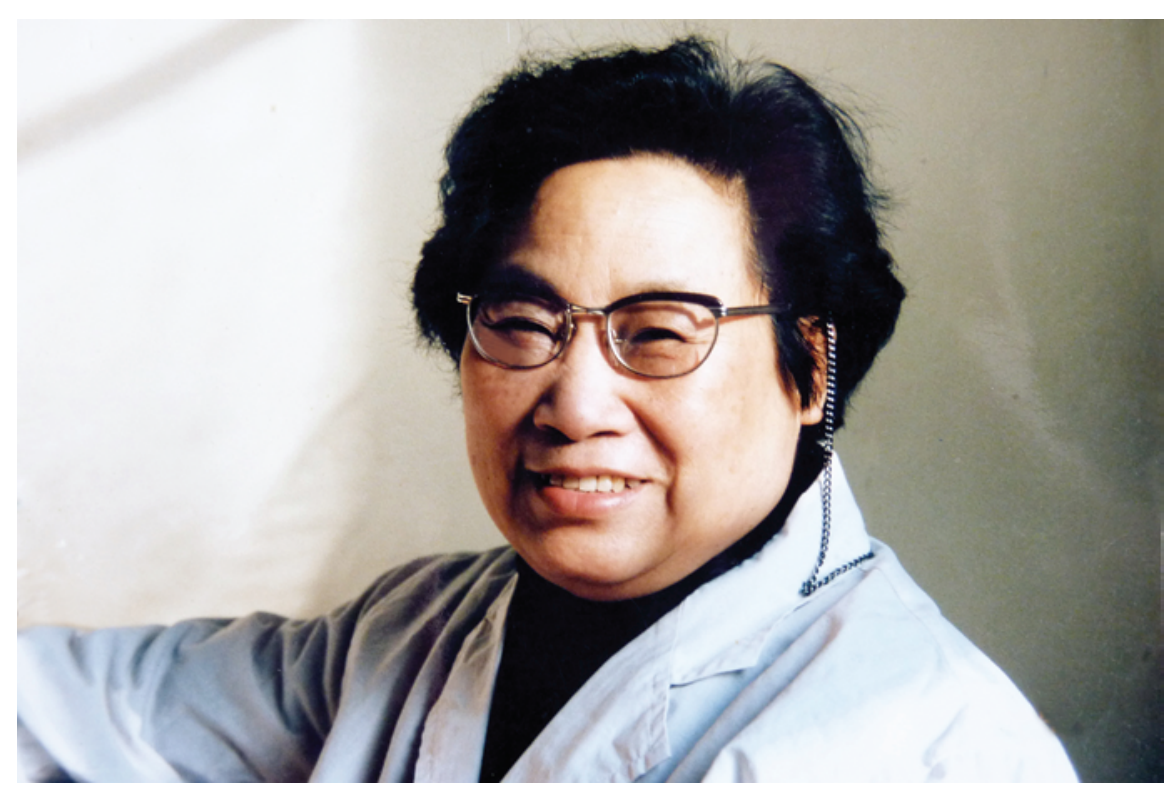

Figure 1

Youyou Tu, winner of the 2011 Lasker DeBakey Clinical Medical Research Award. 


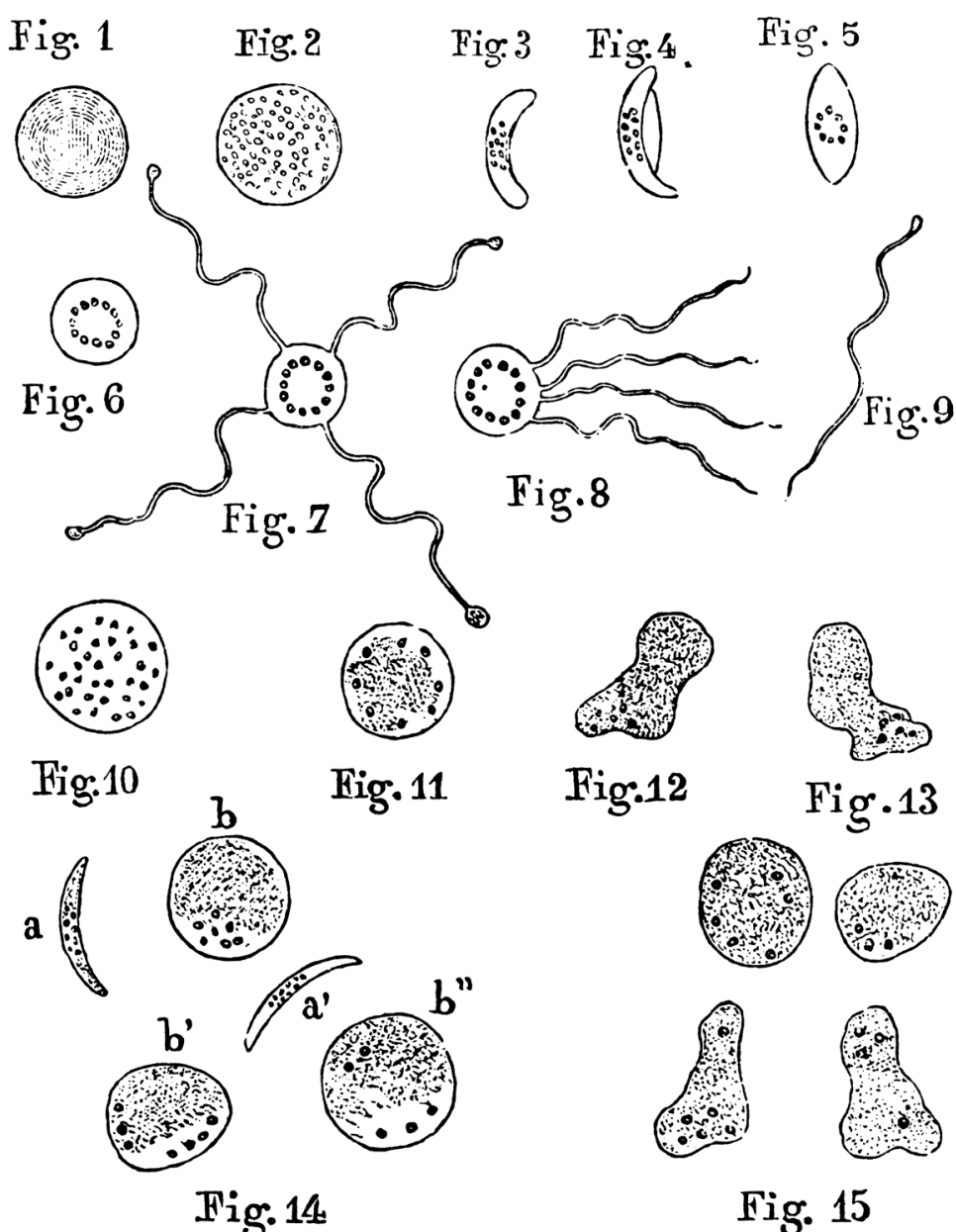

Figure 2

Drawings of malaria parasites by Charles Laveran. Reproduced from Bulletin de l'Académie Nationale de Médecine (4).

which results in anemia due to the destruction of rbc and large amounts of free hemoglobin released into circulation.

The first treatment for malaria was quinine - from the bark of the cinchona tree. Peruvians had long used tinctures of cinchona to treat malarial fevers, but it was in 1820 that the active ingredient, quinine, was extracted and isolated from cinchona bark by the French chemists Pierre-Joseph Pelletier and Joseph Bienaimé Caventou (7). Quinine remained the drug of choice until the 1940s; however, when the Japanese seized Dutch cinchona plantations in Java during World War II, there was a vast quinine shortage, particularly in China (7). The next effective malaria treatment, chloroquine, was synthesized in 1934 at the Bayer laboratories, but it was shelved for at least a decade due to excess toxicity in humans (8). During
World War II, US government-sponsored clinical trials showed that chloroquine was an effective antimalarial drug, and therefore it was introduced into clinical practice in 1947 for both the treatment and prevention of malaria (8). Unfortunately, chloroquine-resistant Plasmodium parasites emerged, and that is where our story meets its heroine.

\section{Unique environment for a unique scientist}

Youyou Tu was born in Ningbo, a city on the east coast of China, at the end of 1930. Her father was a staff member in an organization, while her mother stayed home to tend to Tu and her four brothers. She received four years of training at the School of Pharmacy, Beijing Medical College, between 1951 and 1955. In those days, most graduates with superior aca- demic credentials like Tu were assigned to work in the research institutes due to the huge demand for medical research professionals in China. "I was chosen to work in the Institute of Materia Medica, Academy of Traditional Chinese Medicine, and have worked at the institute for over 55 years since then," Tu told the JCI. Her responsibility within the organization evolved from bench scientist to associate professor, professor, and then department head. "Both of my parents received a good education, and I always feel lucky and thankful to my parents for their dedicated support for me to complete my education. My father has some knowledge in traditional Chinese medicine, and he gave me plenty of backup when I selected medicine as my career," Tu noted.

Tu's interests in traditional Chinese medicines were partially stimulated by the environment in which she grew up: "In my childhood, a lot of folk recipes were used for the treatment or relief of some disease symptoms, and some of them were quite effective." She went on to note, "However, my primary influence was probably from Professor Lin Qi Shou, one of the most respected phytochemists in China, whom I met at the university. He passed his knowledge and in particular his diligence in herb research to the young generation. It was then my interests turned into motivation."

After joining the Institute of Materia Medica, Tu attended a 2.5-year full-time training course on the use of traditional Chinese medicines. She noted, "This systemic training really equipped me with a unique, integrated, and philosophic knowledge encapsulating the modern pharmaceutical sciences and traditional Chinese medicine." At the institute, the primary goals were to search for medications to treat various diseases, in particular those that remained uncured by Western medicines. Communist China was at the height of the Cultural Revolution (1966-1976), a period that was marked by chaos and in which scientific research had all but stagnated. The institute where Tu was working employed not only traditional Chinese practitioners and historians, but also chemists, pharmacologists, and other medical doctors. Tu's workplace was a unique setup specific to institutions in China where scientists worked side by side with historians (9).

\section{Project 523}

During the Vietnam War (1955-1975), the Chinese government supported Vietnam, 


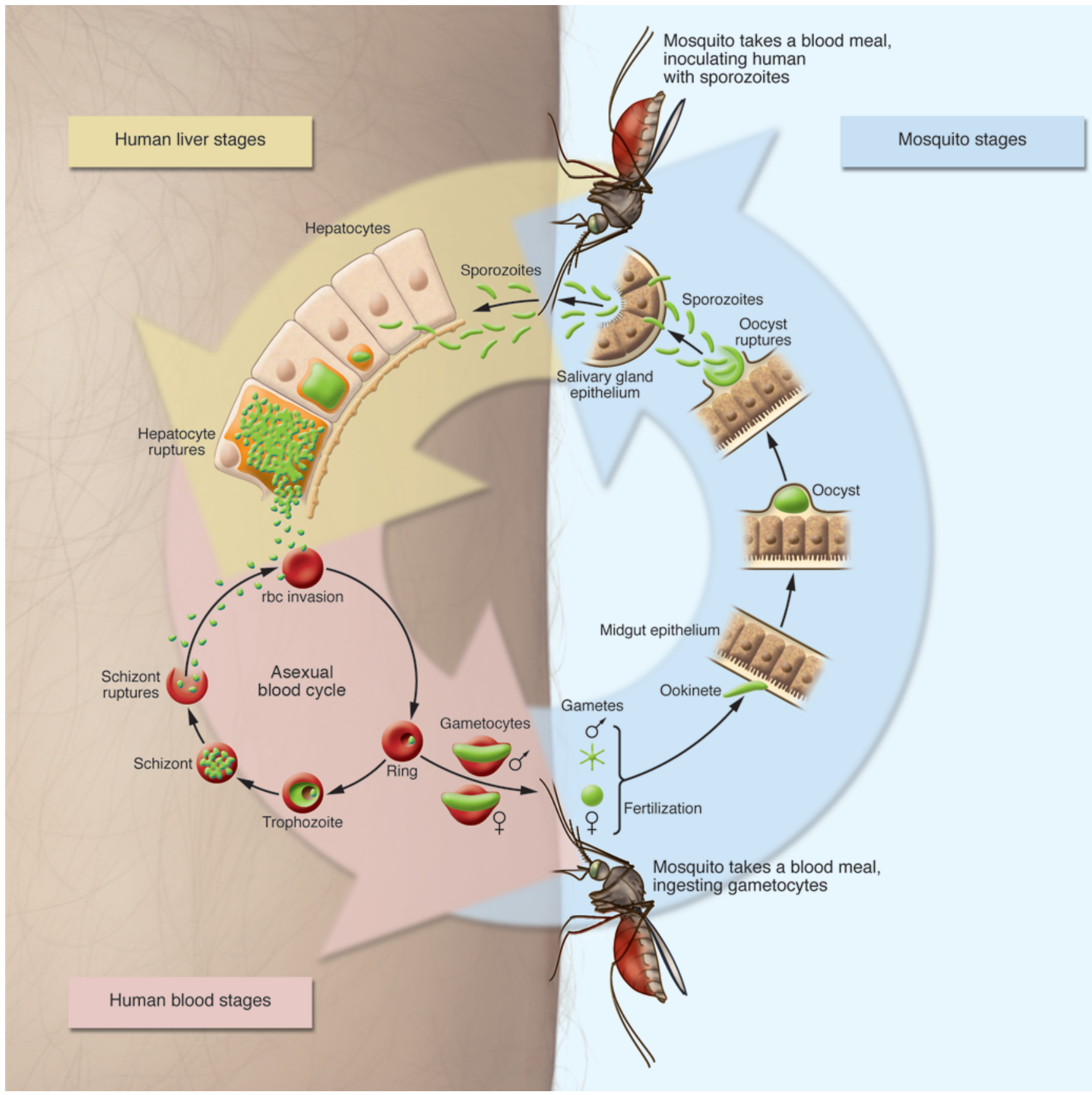

\section{Figure 3}

The life cycle of malaria-causing Plasmodium parasites. Upon inoculation by an Anopheles mosquito into the human dermis, elongated motile sporozoites are injected into the skin or directly into the bloodstream. Sporozoites migrate to the liver and infect a small number of hepatocytes. A single sporozoite gives rise to tens of thousands of asexual parasites called merozoites. After approximately one week, merozoites exit the liver into the bloodstream, leaving no residual parasites in the liver. Merozoites entering the bloodstream begin a cycle of erythrocyte invasion, replication, erythrocyte rupture, and merozoite release that repeats approximately every 48 hours. Symptoms of malaria only occur during the blood stage of infection. A small percentage of blood-stage asexual parasites convert to sexual forms, or gametocytes, which can re-infect mosquitoes. Image modified from ref. 5.

then at war with the United States. North Vietnamese leaders, suffering equally heavy losses of soldiers due to malaria as from warfare, asked the government of China to urgently find malaria remedies. A clandestine venture, named Project 523 for the day it was established-May 23, 1967 - set out to develop antimalarial therapies (10).
The covert nature of the project created a milieu in which few scientific papers were published, with the earliest ones inaccessible outside of China. Many other details 


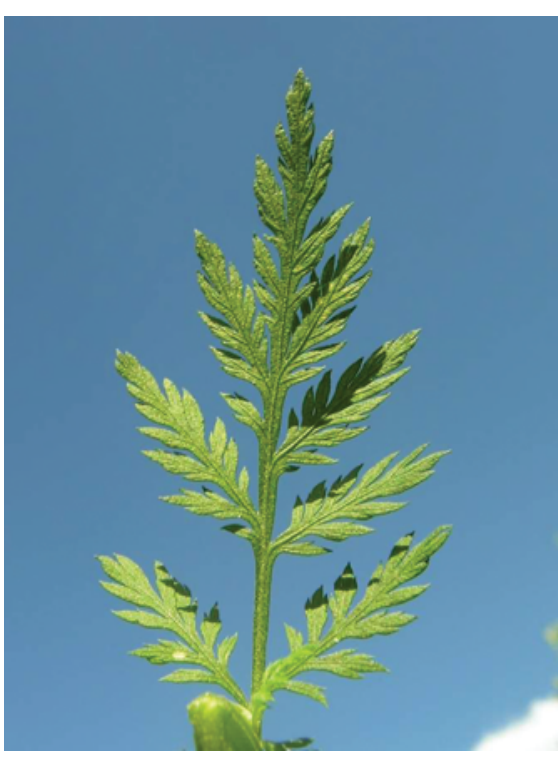

Figure 4

Artemisia annua L., or sweet wormwood.

about the project are still cloaked in secrecy (8). However, it is known that in early 1969 , Tu was named the head of the team at her Institute. Tu recalls, "The reason I was appointed as the head of the Project 523 research group was partially due to the fact that I received training on both modern pharmaceutical sciences and traditional medicine and due to my hard work.”

\section{Parsley, sage, rosemary, and qinghao}

Tu and her team combed ancient texts and folk remedies for possible malaria remedies: "Most ancient texts were readily available from the institute, some from the university libraries and even the museums. Traditional Chinese medicine is a real 'treasure house." The team collected 2,000 recipes from 640 herbs, which Tu then narrowed down to a few promising candidates.

One of the most promising of the candidates was qinghao (Artemisia annua L., or sweet wormwood; Figure 4), as for thousands of years Chinese herbalists had used qinghao as a remedy for various illnesses (10). The earliest written record of qinghao use, written on a piece of silk unearthed from the Mawangdui Han Dynasty tombs (168 BCE), described its use as a treatment for hemorrhoids. Another ancient text, Compendium of Materia Medica by Li Shizhen (1518-1593) during the Ming Dynasty, specifically described qinghao as a remedy for fever (9).
Two years into Project 523, Tu's team had winnowed their list to 380 extracts from 200 different herb preparations, which they then assessed to see whether they could clear P. berghei from the bloodstream of infected mice. Tu recalls, "It was a very laborious and tedious job, in particular when you faced one failure and another. This was the most challenging stage of the project. However, we did receive constant and consistent motivation (verbal, not material), including encouraging comments in a telegraph instructed by the most respected Premier Zhou Enlai.”

Several of Tu's preparations were based on extracts from qinghao, and in 1971, after testing formulations prepared using a variety of methods, including boiling and ethanol extraction, Tu realized that some preparations from Artemisia plants had activities against rodent malaria: "Qinghao extract was proven effective in suppressing parasitemia in the P. berghei-infected mice in the initial studies (68\% inhibitory rate), but the results were inconsistent in the repeated tests. This puzzled us. Qinghao would be a potential candidate only if the original observations could be repeated."

After the confirmatory experiments gave inconsistent answers, Tu started reviewing the literature again. "I had to reconsider all possible alternative sources and approaches and carry out a large number of experiments. Unfortunately, most of these experiments yielded negative results." But then Tu found one sentence in the Chinese medical archive that caught her attention. It was found in Handbook of Prescriptions for Emergencies by the physician Ge Hong (284-363): "Another recipe: qinghao, one bunch, take two sheng [2.2 liters] of water for soaking it, wring it out, take the juice, ingest it in its entirety" (9). This triggered a new idea: the heating involved in the conventional extraction step might have destroyed the active components, and lower temperatures during the extraction process might be necessary to preserve its activity. Tu says it was a revelation: "With this assumption in mind, I redesigned the extraction process using solvents with a low boiling point and also separated the extracts into acidic and neutral by treating them with either acidic or neutral aqueous solutions during the process. We finally obtained extract number 191 from the neutral solvent, which was found to be $100 \%$ effective against parasitemia in both P. berghei-infected mice and P. cynomolgi-infected monkeys on October 4th, 1971." This finding symbolized the breakthrough in the discovery of qinghaosu (qinghao is the Chinese name of Artemisia annua L., and su means "basic element"). Qinghaosu was later called artemisinin.

Despite the early help Tu had from the ancient texts, there were a number of steps she had to sort out on her own. Qinghao is a general name of a category of herbs in traditional Chinese medicine, but there are six types of herbs within this category, and each contains different chemical components with various degrees of efficacy in treating malaria. Ge Hong's handbook did not specify which type of qinghao should be used for treating fever. Tu says, "We found out that only Artemisia annua L. extract has the antimalaria effect. We also did some evaluations correlating the geographical locations where Artemisia annua L. grows with the richness of artemisinin." In addition, Ge Hong's handbook was not specific about which part of the plant - roots, stems, or leaves - was the effective part; Tu came to discover that only fresh leaves contain artemisinin. Tu's team also had to determine the best season for collection, finding that only leaves collected before floral initiation contain sufficient artemisinin. Given these idiosyncrasies in the process, it is amazing that Tu persevered. She notes, "All these uncertainties caused confusion and inconsistent results in our early stages of research.”

When I asked how she felt when she realized what she had discovered, Tu replied, "It is not something I can easily describe, especially after facing so many failures. It almost made me feel that it's not true, which then turned into excitement that what I found is a remedy for the treatment of malaria and not just a finding for a research paper."

Project 523 was proving successful. Tu notes that she initially only had four people in her research group, though more filtered in as the project progressed. By her analysis, it was not an expensive study to conduct at the time, and given that it was a governmental project, funding was guaranteed on an as-needed basis. Tu notes, "We were not allowed to disclose any information beyond the 523 project teams. However, within and between the 523 project groups, we shared the information freely and in fact communicated the research results immediately. That prompted many institutes/researchers to participate in the 
research on qinghao extract after my report on its efficacy in March 1972.”

\section{But would it work in humans?}

During the Cultural Revolution, there were no facilities for performing trials of new drugs, so in order to determine whether artemisinin could be used safely in humans, Tu and her colleagues bravely acted as the first group of volunteers and took the new extract themselves: "It is true that three of my team members including myself pioneered in taking the first doses of the extract for the reasons that (a) we wanted to move on to clinical trials quickly and therefore needed answers as to its safety regime and (b) there was no formal procedure at that time in China regarding safety and clinical evaluation. The only confidence we had was based on the fact that the herb had been used as a medicine in ancient China. Truly, the real motivation was our eagerness to prove the clinical efficacy of this rarely found remedy as soon as possible."

After their first successful safety experiments on themselves, Tu and her team went to Hainan Province, an island off the southern coast of China, to verify the efficacy of the extract clinically and carried out antimalarial trials with 21 patients infected with P. vivax and/or P. falciparum. These experiments produced encouraging feedback, achieving a rapid disappearance of fever and parasites from the blood as compared with the control group using chloroquine (10).

The next step was to isolate the active ingredients, as at the time, artemisinin's active substance had not yet been isolated in its pure form, nor was its structure known. They succeeded in identifying the colorless crystalline substance with a molecular weight of $282 \mathrm{Da}$, with a molecular formula of $\mathrm{C}_{15} \mathrm{H}_{22} \mathrm{O}_{5}$. In $1973 \mathrm{Tu}$ also synthesized dihydroartemisinin, to prove that the chemical structure had a hydroxyl group; she was unaware that the chemical substance she had just produced would later be found to be more effective than the natural compound. In addition, the hydroxyl group provided better opportunities for creating derivatives with potential activity. The stereochemistry and structure of artemisinin were later determined in 1975 with the assistance of the Institute of Biophysics, Chinese Academy of Sciences, as that of a sesquiterpene lactone (11).

Subsequent clinical trials on additional malaria-infected patients confirmed that the crystal they had isolated delivers the antimalarial blow. Tu mentioned that they weren't looking to determine the exact mechanism of action, just to find an effective drug: "What we noticed from the early-stage discovery was that the patients cleared their high fevers, which was the first positive sign of the alleviation of malaria symptoms, and more importantly, we noticed the disappearance of parasites from the blood samples, which was consistent with the results from our earlier animal studies; at that point, we concluded that the medicine cured the disease rather than just eliminating the symptoms. From our observation, artemisinin destroyed malaria species at all stages of the parasite's life cycle." There is still some controversy on the mode of action of artemisinins, and although a number of potential targets have been proposed, the mechanism underlying artemisinin's actions remains to be entirely elucidated.

\section{It is true that three of my team members including myself pioneered in taking the first doses of the extract.}

Many scientists from other institutes across China worked to improve the extraction procedures and conduct subsequent clinical trials (10). Tu notes, "Nothing was disclosed [publicly] until 1978, when the first official report on the history of the artemisinin discovery was published by the Guangming Daily. My name and my institute then became known to the public as the scientists who discovered the medicine." The first English-language report about artemisinin was in December 1979 (12). By that point, artemisinin and its variants had been tested in more than 2,000 patients, some of whom had chloroquine-resistant $P$. falciparum malaria infections.

\section{Bringing artemisinin to the world}

In the mid-1970s, Guoqiao Li (Guangzhou College of Traditional Chinese Medicine) performed clinical trials with artemisinin and its derivatives. Artemisinin-based compounds were shown to be more therapeutically effective than the standard drugs, such as chloroquine and quinine (10). Extra endeavors of Project 523 also led to the creation of other synthetic antimalarial drugs, including pyronaridine in 1973, lumefantrine (benflumetol) in 1976, and naphthoquine in 1986 (10). Some of these compounds are currently being tested as partner drugs in artemisinin-based combination therapies (ACTs).

After the first set of large-scale trials, Keith Arnold (Roche Far East Research Foundation, Hong Kong) joined forces with $\mathrm{Li}$ to test artemisinin, and two years later, they published the first high-profile clinical trial of artemisinin (13). Later, they compared artemisinin alone with the known antimalarial agents mefloquine and Fansidar and showed synergistic effects with no further side effects. After years of clinical trials of various agents, ACTs are now generally considered as the best current treatment for uncomplicated falciparum malaria.

Tu says that she was proud of the potential therapeutic implications of her work, but stayed out of most of the clinical trials in order to focus on the science: "[Happily,] a lot of people contacted me purely for academic interests rather than commercial interests. I have continuously focused my interests and research on the development of more potent derivatives and application of artemisinin medicines for the treatment of other diseases." But she did have a chance to speak with a special malaria patient who benefitted from her work: "Professor Xie Zhongwan, one of my close colleagues, unfortunately contracted malaria in his late 70 s during a trip to investigate rare herbs in the provinces in south China. He rapidly recovered from the disease after administration of artemisinin tablets.”

Unfortunately, pockets of artemisininresistant malaria have appeared, and $\mathrm{Tu}$ is concerned: "Like other scientists in the field, I was extremely concerned and anxious when I saw the recent reports on the emergence of artemisinin-resistant malaria. The WHO has made a correct and timely strategic decision to suspend use of the single active dosage form for the treatment of malaria in order to combat the evolution of resistance. Mass administration of artemisinin as a preventive medicine reported on several occasions did cause 
me serious concern, as it is a potential cause of drug resistance, and I am hopeful that the international community will take a responsible approach in standardizing malaria treatment protocols as well as stopping any kind of abuse in the use of artemisinin medicines.”

\section{The Lasker luster}

$\mathrm{Tu}$ notes that artemisinin is a gift from the "treasure house" of the traditional Chinese medicine crystallized through the hard work of many scientists. But her hard work and dedication is itself a gift of life to millions the world over. She is elated but circumspect about the honor of being awarded the 2011 Lasker DeBakey Clinical Medical Research Award, saying, "[When I heard I won, I had] multiple reactions: I was extremely honored as the winner of the renowned Lasker Award; humbled as an ordinary phytochemist without rich international publications; proud as a Chinese scientist with a discovery based on old Chinese medicine recognized by international science circles."

$\mathrm{Tu}$ finished the interview by noting that her parents were central in setting her down the right path from birth, "My [first] name, Youyou, was given by my father, who adapted it from the sentence “呦呦鹿鸣, 食野之蒿” translated as ‘Deer bleat "youyou" while they are eating the wild Hao' (note: 'Hao' in this context means qinghao) in the Chinese Book of Odes. How this links my whole life with qinghao will probably remain an interesting coincidence forever.”

\section{Ushma S. Neill}

1. Joy D, et al. Early origin and recent expansion of Plasmodium falciparum. Science. 2003; 300(5617):318-321.

2. Cox FEG. History of human parasitology. Clin Microbiol Rev. 2002;15(4):595-612.

3. Nobelprize.org. The Nobel Prize in Physiology or Medicine 1902: Ronald Ross. Accessed August 4,
2011. http://nobelprize.org/nobel_prizes/medicine/ aureates $/ 1902$

4. Laveran CLA. Note sur un nouveau parasite trouvé dans le sang de plusieurs malades atteints de fièvre palustres. Bull Acad Natl Med. 1880;9:1235-1236.

5. Greenwood BM, et al. Malaria: progress, perils, and prospects for eradication. J Clin Invest. 2008; 118(4):1266-1276.

6. Wellems TE, Hayton K, Fairhurst RM. The impact of malaria parasitism: from corpuscles to communities. J Clin Invest. 2009;119(9):2496-2505.

7. Burns WR. East meets West: how China almost cured malaria. Endeavour. 2008;32(3):101-106.

8. Jensen M, Mehlhorn H. Seventy-five years of Resochin in the fight against malaria. Parasitol Res. 2009; 105(3):609-627.

9. Hsu E. Reflections on the "discovery" of the antimalarial qinghao. Br J Clin Pharmacol. 2006; 61(6):666-670.

10. Cui L, Su XZ. Discovery, mechanisms of action and combination therapy of artemisinin. Expert Rev Anti Infect Ther. 2009;7(8):999-1013.

11. Collaboration research group for Qinghaosu. A new sesquiterpene lactone - Qinghaosu [in Chinese]. Kexue Tongbao. 1977;3:142.

12. [No authors listed]. Antimalaria studies on Qinghaosu. Chin Med J (Engl). 1979;92(12):811-816.

13. Jiang JB, Li GQ, Guo XB, Kong YC, Arnold K. Antimalarial activity of mefloquine and qinghaosu. Lancet. 1982;2(8293):285-288. 